hen?; (2) wie gehen Mitglieder damit um, dass eine Parteimitgliedschaft „von der Wiege bis zur Bahre" eine für die moderne Biografie unwahrscheinliche Erzählung darstellt?; (3) in welchem Verhältnis stehen politische Beratung und Beratene?" (S. 151) verbindet Siri empirische Forschung und Theoriedebatten der Soziologie. Sie nimmt mit Wahlkampf und Kampagnen, Programmen, Erzählung der Mitgliedschaft und politischer Beratung organisatorische Gegenwarten in den Blick, die jeweils eigenlogisch funktionieren und miteinander oft weniger zu schaffen haben, als mediale und wissenschaftliche Beobachter meinen.

Jasmin Siri leistet mit dem Perspektivenwechsel von der „Fiktion einer identen ParteienPerson“ hin zu Parteien als paradoxen Organisationen einen originären wissenschaftlichen Beitrag. Aus der Widersprüchlichkeit der Anforderungen durch Legitimation/Partizipation und Effektivität/handlungsbefähigende Geschlossenheit kann sie ableiten, dass Parteienkritik zwar ihren Ursprung in den unerfüllbaren Erwartungen identitärer Demokratie hat, aber als Motor permanent zu Erneuerung und Reform herausfordert. „Die paradoxe Wirkung der Kritik an den Parteien besteht in ihrer Funktionalität für die Reproduktion der Organisationen und des politischen Systems. Die Kritik ermöglicht das Präsenthalten demokratischer Ideale, die Krisendiagnostik das Anstoßen von Reformen“" (S. 254).

Da es kaum Arbeiten über Wahlgesetze und die unterschiedlichen Satzungen der Parteien gibt, öffnet sich mit der Aufmerksamkeit für Organisation ein neues Forschungsfeld. Mit dem Blick auf die Aufgaben von Parteien und deren Gemeinsamkeiten wird es möglich, über Einzelstudien zu CDU, SPD, FDP, Grünen, Linken und Piraten hinaus zu untersuchen, unter welchen Wahlgesetzen und mit welchen Satzungen sich die widersprüchlichen Anforderungen wenigstens ansatzmäßig vereinbaren lassen. Den Praktikern in den Parteien dürfte der Hinweis auf die strukturellen Brüche helfen, die widersprüchlichen Erwartungen als nicht nur individuelles Problem zu begreifen. Beratern in und außerhalb der Parteien könnten die systematische Darstellung des nicht zu unterschätzenden Wandels der Parteiorganisationen durch immer mehr Kontakte mit den Massenmedien und wachsenden Bedarf an Beratungskommunikation nützen. Die nicht einfache Lektüre des Textes lohnt sich und lädt den interessierten Leser ein zu prüfen, welche Bedingungen, Erwartungen und Aporien in die eigenen Analysen einfließen.

Ingrid Reichart-Dreyer

\title{
SPD: Personen und Programme am Ende der Ära als Volkspartei
}

Reinhardt, Max: Aufstieg und Krise der SPD. Flügel und Repräsentanten einer pluralistischen Volkspartei, Nomos Verlagsgesellschaft, Baden-Baden 2011, 628 Seiten, € 99,-.

Sachs, Matthias: Sozialdemokratie im Wandel. Programmatische Neustrukturierungen im europäischen Vergleich, VS Verlag für Sozialwissenschaften, Wiesbaden 2011, 381 Seiten, € 39,95.

Die gute alte SPD, die Sozialdemokratie als Volks- und Mitgliederpartei, auch als kulturelles Milieu und als soziale Bewegung wird - 150 Jahre nach ihrer Gründung - immer mehr zu einem geschichtlichen Phänomen, versinkt gleichsam im Horizont der Historie. Je mehr jedoch diese Partei zur Vergangenheit wird, desto intensiver befassen sich Historiker, Politikund Sozialwissenschaftler mit ihr, wird gefragt, ob die Solidargemeinschaft, die Volkspartei, 
die Mitgliederpartei revitalisiert werden könne oder ob sie endgültig im Museum ihren Platz findet. In diesen Kontext gehören die hier anzuzeigenden Monografien, beides Dissertationen. Nicht ganz zufällig wurden beide Autoren von der Friedrich-Ebert-Stiftung in ihrer wissenschaftlichen Arbeit gefördert.

Matthias Sachs untersucht die Programmdiskussion in drei sozialdemokratischen Parteien im Zeitraum von Mitte der 1980er Jahre bis circa 2007 - und zwar die SPD 1984 bis 2007 (also bis zum Hamburger Programm), die britische Labour Party von 1987 bis 2005 und die niederländische Partij van de Arbeid 1986 bis 2005. Seine Fallauswahl begründet Sachs wie folgt: Die drei Parteien agierten vor einem je unterschiedlichen gesellschaftlichen und systemischen Hintergrund und repräsentierten zwei sehr unterschiedliche Modelle sozialer Demokratie beziehungsweise sei eine Variante zwischen beiden angesiedelt. So bestehe bis heute in Deutschland eine in ,großen Teilen traditionelle und konservativ-korporatistische Sozialdemokratie“, während sich in Großbritannien in der Entwicklung von Old zu New Labour eine „sich neo-liberalen Ideen nicht verschließende“ Sozialdemokratie herausgebildet habe. In den Niederlanden sei die Partij van de Arbeid zwar liberaler geworden, doch versuche sie als Regierungspartei im Kontext des Polder-Modells „im Konsensverfahren die freien Kräfte des Marktes eindeutiger zu kanalisieren und einzuhegen“, stehe also zwischen SPD und Labour.

Eine erkenntnisleitende Fragestellung ist vom Autor leider nur angedeutet, nicht systematisch formuliert, elaboriert und operationalisiert. Vielmehr bleibt Sachs recht allgemein, fragt „inwiefern die drei Parteien als gestaltende Kräfte neuer sozialdemokratischer Modelle in Erscheinung getreten sind, beziehungsweise ob in den untersuchten Programmdebatten eine zukunftsfähige Weiterentwicklung sozialdemokratischer Programmatik erkennbar geworden ist". Ansonsten legt Sachs die Programme der untersuchten Parteien nach vier Indikatoren, nach vier Herausforderungen unterschieden nebeneinander, nämlich die programmatischen Antworten auf die Globalisierung, auf die Informatisierung (verstanden als Einführung neuer Technologien unter Berücksichtigung der humanen und sozialen Folgen), auf die Individualisierung und auf neue Formen der Politik. In jedem Kapitel, in dem von einer der drei Parteien gehandelt wird, werden zudem die jeweiligen Partei- und Wahlsysteme unter der Frage dargestellt, inwieweit sich die drei Sozialdemokratien in ihren Programmdebatten an diese vorgegebenen Strukturen angepasst haben beziehungsweise anpassen mussten (zum Beispiel Rücksichtnahme auf künftige Koalitionspartner).

Der Autor stellt in seinem Text die These auf, dass Programme gerade für sozialistische und sozialdemokratische Parteien „existentiell und besonders bedeutsam“ seien. Er begründet dies auch ansatzweise. Doch hätte die Untersuchung erheblich gewonnen, wenn Sachs hier noch intensiver auf die Funktion von Programmen eingegangen wäre, etwa auf die Integration der verschiedenen Flügel einer Partei durch innerparteiliche Programmdiskussionen, Missbrauch und Manipulation der Programmdebatten, indem Parteiintellektuelle durch sie still gestellt und von der eigentlichen Politik ferngehalten werden, Relevanz beziehungsweise Irrelevanz von Programmen für Wähler. Und: Warum sind Programme für sozialistische und sozialdemokratische Parteien eigentlich wichtiger als für andere Parteien? Überhaupt arbeitet der Autor an verschiedenen Stellen seines Werkes mit einem engen, verkürzten Programmbegriff, so wenn er schreibt, der Fokus seiner Darstellung liege auf den beiden im Untersuchungszeitraum verabschiedeten Grundsatzprogrammen der SPD. Zwar zieht Sachs für seine Darstellung des Berliner und Hamburger Programms nicht nur Entwürfe heran, sondern auch Arbeitshefte, „Papers“ aller Art, Zeitschriftenbeiträge, Sammel- 
hefte, Impulspapiere, Berichte und Kommentare. Doch verfehlt er damit die eigentliche, die Partei fast „umstürzende“ programmatische Änderung in diesem Zeitraum, nämlich die Agenda 2010. Regierungspolitik und Regierungshandeln dürfen nicht aus einer Analyse der Programmentwicklung ausgeblendet werden.

In der Zusammenfassung der Monografie „werden die in den (...) Kapiteln gemachten Feststellungen bezüglich der Wahl- beziehungsweise Parteiensysteme nebeneinander gelegt, und die jeweiligen Auswirkrungen auf die Programmarbeit der Parteien miteinander verglichen“. Dabei überrascht es nicht, dass Sachs zu dem Ergebnis kommt, dass die drei sozialdemokratischen Parteien in unterschiedlichem Maße ihre politische Konkurrenz beobachten und bei der Formulierung ihrer eigenen Programmatik berücksichtigen müssen.

Ein neues sozialdemokratisches Modell ist dabei nicht konzipiert worden. Auch in der Zusammenfassung bedient Sachs sich der vier von ihm eingeführten Indikatoren beziehungsweise Herausforderungen, bleibt stark in der Deskription, fragt analytisch aber nur andeutungsweise, warum bei bestimmten Antworten auf die Herausforderungen eine gewisse Kongruenz besteht, bei anderen hingegen nicht.

Das Buch von Max Reinhardt vereint zwei Darstellungen, die - wie der Betreuer der Dissertation in seinem Vorwort treffend schreibt - üblicherweise getrennt sind. Die eine Darstellung enthält eine Geschichte der innerparteilichen Richtungskämpfe und Strömungen in der SPD seit 1945, die andere widmet sich dreizehn Führungspersönlichkeiten der Partei, dreizehn ausgewählten Akteuren, die in ausführlichen Interviews befragt worden sind. Reinhardt geht von der Frage aus, ob sich die parteiflügel- und milieuübergreifende Repräsentationsfähigkeit der SPD und ihrer Spitzenpolitiker gewandelt hat. Die Hypothese der Arbeit lautet, dass die heutige SPD insbesondere seit ihrem Regierungsantritt 1998 nicht nur Wählerverluste hinnehmen musste, „sondern das Ergebnis eines Wandels in Folge von innerparteilichen Machtkämpfen und oftmals autoritär geführten Ausgrenzungshandlungen ist". Die SPD, so der Autor, könnte dabei ihren Charakter als Volkspartei verloren haben, der in ihrer milieuübergreifenden Integrations- und Repräsentationsfähigkeit bestanden hatte.

Die Analyse innerparteilicher Machtkämpfe bietet für den Sozialwissenschaftler eine faszinierende Thematik, geht es doch hier um informelle, häufig verborgene und im Hintergrund sich abspielende Auseinandersetzungen und Konflikte. Reinhardt schließt in seiner detaillierten und kenntnisreichen Untersuchung an ältere Arbeiten an, so an die von Ferdinand Müller-Rommel von 1982 über innerparteiliche Gruppierungen in der SPD. Er ist in seiner Darstellung um begriffliche Präzision bemüht, unterscheidet anhand des Institutionalisierungsgrades und der Personalisierung zwischen Strömungen, Gruppierungen, Flügeln, Faktionen und Fraktionen. Die innerparteilichen SPD-Flügel sind für ihn Faktionen. In der chronologisch angelegten Beschreibung der Flügelkämpfe arbeitet Reinhardt immer wieder die Heterogenität der verschiedenen Faktionen heraus. Der Text basiert im Wesentlichen auf einschlägiger Sekundärliteratur. Warum der Autor nicht schon in diesem Teil seiner Arbeit die Interviews wenigstens mit den dreizehn Spitzenpolitikern intensiver und nicht nur sporadisch herangezogen hat, bleibt für den Leser rätselhaft. Gerade die biografischen Abschnitte und Elemente zeugen nicht nur von ungeheurem Fleiß des Autors, sondern geben grundlegendes Quellenmaterial ab. Dies gilt auch für circa 400 Kurzbiografien, die sich im Anmerkungsapparat finden, in vielen Fällen recht umfänglich und detailreich sind und durchaus Material über die Flügelbildung der SPD enthalten.

In der Auswertung der dreizehn Biografien sozialdemokratischer Spitzenpolitiker kommt Reinhardt zu dem Ergebnis, dass die Repräsentation der SPD sich auf zwei Typen verengt 
habe, nämlich auf den dominanten, kleinbürgerlichen, leistungsorientiert-ständisch denkenden „Neuen Managertypus“ mit einem Gesellschaftsbild von individualisierten Arbeitnehmern (zu dem er Sigmar Gabriel, Thomas Oppermann, Olaf Scholz, Frank-Walter Steinmeier und Brigitte Zypries rechnet) und auf den modernisierten gewerkschaftsnahen „Arbeitnehmertypus" mit dem skandinavischen Gesellschaftsbild eines solidarischen Arbeitnehmers (Andrea Nahles). Wo die Sympathien des Verfassers liegen, ist offenkundig: bei der linken Faktion. Allerdings bedauert er, dass die heutige SPD-Linke keine systemüberwindenden Positionen vertrete und letztlich zu einem Mitte-Links-Bündnis geworden sei. Ihr fehlten die entschiedenen Sozialisten, die Kurt Schumacher noch integriert habe. Mit besonderer Zuneigung behandelt Reinhardt sein Idol Peter von Oertzen, erweckt fast den Eindruck, als seien dessen rätesozialistische Positionen zeitweilig in einer Faktion der SPD vertreten worden.

Abschließend stellt Reinhardt fest, dass die SPD heute wirtschaftsliberal verengt sei, ihr rechter Flügel die Partei dominiere. Er fordert, dass die SPD wieder milieuübergreifende und -integrierende Volkspartei werden müsse. Diese Forderung bleibt recht voluntaristisch, struktureller Wandel wird nur unzureichend bedacht: Veränderungen zwischen sekundärem und tertiärem Sektor; Ende der Arbeiterkulturbewegung und damit des entsprechenden Milieus; Ende des Organisationsnetzwerkes, das die Partei und ihre Kultur- und Freizeitvereine einst zusammenhielt. Das Ende der Volksparteien, so, wie wir sie aus der Geschichte kennen, dürfte endgültig gekommen sein - und damit auch das der Volkspartei SPD.

Um nicht missverstanden zu werden: Die normativen Positionen und auch Zuspitzungen Reinhardts sind nicht negativ zu kritisieren, vielmehr positiv hervorzuheben. In einem Zeitalter, in dem Doktoranden sich brav an ihre akademischen „Väter" und „Mütter“ anpassen, ist eine Dissertation wie die vorliegende erfrischend, reizt zum Widerspruch oder zur vehementen Zustimmung. Dies spricht für den Autor, Max Reinhardt, und seinen Betreuer, Michael Vester. Was mehr irritiert, ist das Auseinanderfallen des Bandes in zwei Darstellungen.

Peter Lösche

\section{CDU: Strategien der Machterhaltung und innere Spannungsfelder auf dem Weg in die Mitte}

Walter, Franz, Christian Werwath und Oliver d'Antonio: Die CDU. Entstehung und Verfall christdemokratischer Geschlossenheit (Schriftenreihe „Die politischen Parteien der Bundesrepublik Deutschland“), Nomos Verlagsgesellschaft, Baden-Baden 2011, 261 Seiten, € 19,90.

Neumann, Arijana: Die CDU aufLandesebene. Politische Strategien im Vergleich, VS Verlag für Sozialwissenschaften, Wiesbaden 2012, 322 Seiten, $€$ 39,95.

Die Machtstrukturen der Parteien und ihre Strategien im Wettbewerb um Wählerstimmen werden am Beispiel der CDU in zwei neu erschienenen Arbeiten untersucht. Während bei Franz Walter, Christian Werwath und Oliver d'Antonio die Anpassung der CDU an veränderte gesellschaftliche Rahmenbedingungen im Zeitverlauf betrachtet wird, analysiert Arijana Neumann Einflussfaktoren einer erfolgreichen Regierungsübernahme der CDU in den Ländern während der Regierungszeit von SPD und Grünen auf Bundesebene. 